\title{
Evaluation of a Bayesian Model Integration-Based Method for Censored Data
}

\author{
Liping Hou ${ }^{a}$ Kai Wang ${ }^{b}$ Christopher W. Bartlett ${ }^{a}$ \\ a Battelle Center for Mathematical Medicine, The Research Institute at Nationwide Children's Hospital and \\ Department of Pediatrics, The Ohio State University, Columbus, Ohio, and ${ }^{b}$ Department of Biostatistics, College of \\ Public Health, University of lowa, lowa City, lowa, USA
}

\section{Key Words}

Quantitative trait threshold model $\cdot$ Censored data

Simulation P P f framework • Bayesian model integration

\begin{abstract}
Objective: Non-random missing data can adversely affect family-based linkage detection through loss of power and possible introduction of bias depending on how censoring is modeled. We examined the statistical properties of a previously proposed quantitative trait threshold (QTT) model developed for when censored data can be reasonably inferred to be beyond an unknown threshold. Methods: The QTT model is a Bayesian model integration approach implemented in the PPL framework that requires neither specification of the threshold nor imputation of the missing data. This model was evaluated under a range of simulated data sets and compared to other methods with missing data imputed. Results: Across the simulated conditions, the addition of a threshold parameter did not change the PPL's properties relative to quantitative trait analysis on non-censored data except for a slight reduction in the average PPL as a reflection of the lowered information content due to censoring. This remained the case for non-normally distributed data and extreme sampling of pedigrees. Conclusions: Overall, the QTT
\end{abstract}

model showed the smallest loss of linkage information relative to alternative approaches and therefore provides a unique analysis tool that obviates the need for ad hoc imputation of censored data in gene mapping studies.

Copyright @ 2012 S. Karger AG, Basel

\section{Introduction}

Many human clinical disorders have non-random missing data in quantitative phenotypic measurements used in gene mapping studies. Systematically missing data may include, for example, treatment effects. Effective blood pressure medication ensures unreliable measurements of the underlying pathological blood pressure state. Sometimes, a measurement may be confounded with the disease status, such as hearing acuity tests not being applicable to individuals unable to hear. In all such cases, the missing data censor the observed trait distribution. While it is always possible to ignore censoring by, on the one side of the spectrum, continuing to use quantitative values with treatment effects, or, on the other side of the spectrum, removing those subjects from the analysis, these options are expected to reduce gene mapping power in families just as they reduce power and produce bi-

\section{KARGER \\ Fax +4161306 1234 \\ E-Mail karger@karger.ch}

www.karger.com
(C) 2012 S. Karger AG, Basel

0001-5652/12/0741-0001\$38.00/0

Accessible online at:

www.karger.com/hhe
Assist. Prof. Christopher W. Bartlett, $\mathrm{PhD}$

Battelle Center for Mathematical Medicine, The Research Institute

Nationwide Children's Hospital and The Ohio State University

JW3926, 700 Children's Drive, Columbus, OH 43205 (USA)

E-Mail christopher.bartlett@ nationwidechildrens.org 
ased parameter estimates in population-based epidemiology cohorts of unrelated individuals [1].

Many formalized statistical methods to account for censored observations in population-based data exist, though, for family data, there are fewer methods available [2-8]; some methods for censoring assume a survival analysis context, and it is unclear how these methods may be applied in other settings $[2,5]$. Despite the availability of methods in the literature, family-based studies underutilize these options since they are not yet implemented in widely available software, thus promoting the use of ad hoc fixes. Since the most commonly used linkage software packages do not account for censoring using an a priori statistical model, the phenotypic data are sometimes manipulated, most commonly, by converting censored observations to a fixed value chosen by the investigators, with what Tobin et al. [1] call a 'sensible constant' [for examples, see also 9, 10]. The choice of the sensible constant may be motivated by exogenous data (i.e., mean phenotypic value for untreated subjects if treatment effects are the reason for censoring) or one of the many methods for single or multiple imputation not specifically designed for family-based analysis [11, 12].

Here, we evaluate an approach to the analysis of censored data that avoids the need for post hoc sensitivity analysis since the method does not require a sensible constant to be defined [13]. The model was defined with the application of two principles. First, for gene mapping studies in pedigrees, phenotyped relatives may mitigate power loss since shared genetics and shared environment predict missing phenotypes proportional to the magnitude of those shared effects. Second, if the direction of the censoring is known or reasonably inferable, such as treatment effects, that information may also be incorporated into the analysis since it effectively limits the range of possible values for the missing data. Here, we examine the properties of using a quantitative trait threshold (QTT) model applied only to the censored observations. Similar to the concept of the liability threshold model [14] where affected individuals are assumed to have genetic liability beyond an undefined threshold, here we assume that censored individuals have trait values beyond an unspecified threshold with uncertainty in this threshold parameter handled in the model. Then we proceed with the calculation using that threshold model for censored individuals while using a standard quantitative trait (QT) analysis model for the remaining observations when building the likelihood of the pedigree for linkage analysis. These principles were incorporated into the PPL framework for computation [13]. Originally formulated as a Bayesian linkage method (PPL stood for the posterior probability of linkage), it is currently best characterized as a family of related methods that differ only in the addition or subtraction of trait and/or genetic model parameters from a common pedigree likelihood engine that can accumulate evidence for linkage and/or association [15]. For this reason, 'PPL' is not fully appropriate as an acronym, but it is still useful as a unique identifier for the framework. The PPL framework is adaptable for modeling complex disease genetics including - as dictated by the user - linkage, association, joint linkage-association, epistasis, imprinting and sex-specific recombination on either categorical or quantitative traits. It has been successfully applied to a variety of complex gene mapping efforts including diseases such as autism, schizophrenia and specific language impairment [16-19]. By design, the PPL framework directly measures the probability of a trait gene linked to the marker (or location) being evaluated [20]. It is on the probability scale $(0-1)$ and can be readily interpreted as a probability. Characteristics of the PPL, as derived, initially applied, and along with further additional computational enhancements are described elsewhere [20-24].

When analyzing quantitative traits, the PPL is parameterized in the same way as the QT likelihood in the traditional LOD score [25] with trait parameters including one (biallelic) trait allele frequency, three genotypic means and corresponding variances. Transmission parameters are the same as in the traditional LOD score. An additional admixture parameter is included to form a heterogeneity LOD score. These nuisance trait parameters are then integrated out of the likelihood. We have shown that this model is quite robust for oligogenic traits, and a further benefit of this form of likelihood is that it is also essentially 'model free' with respect to the distribution of the trait in the population [26]. This issue is of concern when using some alternative approaches such as variance components (VC) methods, which generally show increased false-positive rates (compared to nominal significance levels) when the underlying normality assumption is violated [27]. Additionally, because all forms of the PPL are inherently ascertainment corrected (see Methods section for details), we do not need to correct for ascertainment-like VC analysis (VCA), and we do not need to specify or estimate population parameters such as the population mean and variance-like pedigree-based regression [28].

The QTT model, as presented in the literature review and specified more in the Methods section, uses the same principles as the PPL QT model [26] but with the addition of a threshold parameter to model the censored data. The 
PPL was previously extended to include a threshold parameter to handle specific forms of censoring and has been applied in several contexts as part of larger hypothesis-driven research questions $[13,18,29]$. Using a series of simulations, we examined the properties of the PPL when analyzing censored data where the missing data point can be reasonably inferred to have a value above or below the unknown censoring threshold based on prior knowledge. Using the QTT model does not require estimation or imputation of the missing phenotypes or the censoring threshold. We also analyzed the same simulated data using a sensible constant approach to empirically evaluate how this common ad hoc method performs compared to the QTT model.

\section{Methods}

In this section, we (1) show the modification of the QT LOD score likelihood that underlies the PPL computation, (2) discuss how the PPL is calculated from the LOD scores and (3) describe the simulation methods for assessing model performance.

\section{Likelihood Formulation}

The pedigree likelihood used as the basis for PPL computation is described in full detail elsewhere [26]. Briefly, starting with the pedigree linkage likelihood that forms the basis of the familiar parametric LOD score, the trait model parameters include a recombination fraction $\theta$, disease allele frequency, a vector of penetrances, $P\left(\mathrm{x}_{\mathrm{i}} \mid \mathrm{g}_{\mathrm{j}}\right)$, and the probability of disease $\mathrm{x}_{\mathrm{i}}$ for person $i$, given the three possible trait genotypes $\left(\mathrm{g}_{\mathrm{j}}\right)$ indexed by $j$. The penetrance function $P\left(\mathrm{x}_{\mathrm{i}} \mid \mathrm{g}_{\mathrm{j}}\right)$ is the link between the genotype and phenotype that is reparameterized to allow the likelihood to handle QT data (the transmission parameters from the linkage likelihood are not changed). For quantitative traits, $P\left(\mathrm{x}_{\mathrm{i}} \mid \mathrm{g}_{\mathrm{j}}\right)$ is replaced with $\varphi\left(\mathrm{X}=\mathrm{x} \mid \mu_{\mathrm{i}}, \sigma_{\mathrm{j}}^{2}\right)$, where $j$ indexes the three genotypic distributions for the trait genotypes $\mathrm{AA}, \mathrm{Aa}$ and $\mathrm{aa}$, and $\varphi$ is the standard normal density. Note that the QT model does not assume normality of the overall observed trait data since it is a mixture of three normal distributions, one per genotype, each weighted by the genotypic frequency. Therefore, this model may imply markedly non-normal (even tri-modal) trait data. In practice, however, for reasonable effect sizes generally encountered in complex disease human genetics and simulated here, this mixture model implies overall trait data that do not grossly depart from normality.

For the QTT model, the underlying QT likelihood is modified differentially. If a person has a QT value, then $P\left(\mathrm{x}_{\mathrm{i}} \mid \mathrm{g}_{\mathrm{j}}\right)=\varphi(\mathrm{X}=$ $\mathrm{x}_{\mathrm{i}} \mid \mu_{\mathrm{j}}, \sigma_{\mathrm{j}}^{2}$ ) - thus the same as in the QT model (previous paragraph). However, if a person is coded categorically as 'censored', then we replace $\varphi\left(\mathrm{X}=\mathrm{x}_{\mathrm{i}} \mid \mu_{\mathrm{j}}, \sigma_{\mathrm{j}}^{2}\right)$ with $\varphi\left(\mathrm{x}_{\mathrm{i}}<t \mid \mu_{\mathrm{j}}, \sigma_{\mathrm{j}}^{2}\right)$ if they are assumed to be below the threshold $t$, and $\varphi\left(\mathrm{x}_{\mathrm{i}}>t \mid \mu_{\mathrm{j}}, \sigma^{2}{ }_{\mathrm{j}}\right)$ if not.

\section{Calculation of the PPL}

All QTT calculations were performed with KELVIN, a package for linkage and association analysis of data sets that can include mixes of pedigrees, trios and case-control data [for an overview of KELVIN and its capabilities, see 15]. In this study, we only employed linkage routines on pedigrees. Briefly, the pedigree likelihood is used to calculate many combinations of trait parameters and heterogeneity LOD scores as a fundamental quantity used for numerical integration over the trait parameters. Numerical integration of the model parameters results in an integrated likelihood ratio (often called the Bayes Ratio, or BR, in the PPL literature) that is input into Bayes theorem to quantify the strength of the linkage evidence on the probability scale. As more data are collected, new results are combined with existing results through a procedure called sequential updating. Sequential updating as more data are added is accomplished by multiplication of the BRs across data sets and recomputation of the PPL [20]. In this way, nuisance parameters are integrated out of the BR independently for each data set and are therefore allowed to vary across pedigrees, which is advantageous for genetically heterogeneous disorders.

In calculating the PPL using the QTT model, the parameter $t$ represents the threshold value, beyond which an observation is considered censored. Analytically, integration over the parameter $t$ has the limits $0 \rightarrow \infty$ if $t$ is assumed to be above the population mean, and $-\infty \rightarrow 0$ otherwise. However, due to the restrictions of the numerical integration required for calculating the PPL, limits are imposed based on the accuracy of the desired result. For approximately normally distributed data, numerical integration from $0 \rightarrow 3$ provides the same answer to within 6 decimal places as numerical integration from $0 \rightarrow 6$ (the same is true when changing the direction of integration from $-3 \rightarrow 0$ and $-6 \rightarrow 0$ ). The prior distribution on $t$ is uniform, the same as in the other model parameters, discussed in more detail for the QT model here [26]. Therefore, $t$ is numerically integrated out of the likelihood with the other nuisance model parameters in the same way. Hence, the potential strength of the QTT model is that the value of $t$ needs to be neither specified in the analysis nor estimated from the data.

\section{Data Simulation}

Simulated pedigree data (phenotypes and genotypes) were generated using the same methods and an extended set of model parameters employed to evaluate the original QT model (no threshold) for the PPL [26]. Briefly, we used SIMNUCLEAR (H.H. Göring) to generate data sets of 5-person nuclear families where the number of families was fixed at different values for each of the ascertainment schemes. The four ascertainment schemes were: families ascertained randomly, or based upon the presence of at least one proband child whose quantitative score exceeded $1 \mathrm{SD}$ (ASC 1), 2 SD (ASC 2) or $3 \mathrm{SD}$ (ASC 3) above the population mean. For each ascertainment scheme, the sample size $\mathrm{n}$ was chosen to ensure the average PPL at a linked marker was not near the probability scale boundaries ( 0 and 1$)$, which could have obscured comparisons. For the four ascertainment conditions random, ASC 1, ASC 2, and ASC 3, the sample sizes were 500, 200, 100, and 50 , respectively.

Marker data were simulated for two independent chromosomes so that it was possible to simulate either a single-locus trait and a null chromosome (to assess performance of the QTT model when there is no linkage) or a two-locus trait model. Linked markers were always $\theta=0.01$ from the disease locus, and in the case of two-locus models, the trait loci were on separate chromosomes and, therefore, unlinked $(\theta=0.5)$. 
The trait genotypic parameter values for the loci were either (1) 'Additive' $P(\mathrm{~A})=0.7, \mu \mathrm{AA}=-0.41, \mu \mathrm{Aa}=0.28, \mu \mathrm{aa}=0.97$, $\sigma^{2} \mathrm{AA}=\sigma^{2} \mathrm{Aa}=\sigma^{2} \mathrm{aa}=0.33$; (2) 'Dominant' $\mathrm{P}(\mathrm{A})=0.5, \mu \mathrm{AA}=$ $-0.26, \mu \mathrm{Aa}=-0.26, \mu \mathrm{aa}=0.77, \sigma^{2} \mathrm{AA}=\sigma^{2} \mathrm{Aa}=\sigma^{2} \mathrm{aa}=0.33$, or $(3)$ 'Null' $\mu \mathrm{AA}=\mu \mathrm{Aa}=\mu \mathrm{aa}$ (no linkage). These additive and dominant models have an effect size of 0.2 (i.e., $20 \%$ of the trait variance is attributable to the locus). Additionally, in the simulated data sets, the residual familial correlation (RFC) from polygenes was varied from 0 to 0.4 in increment of 0.1 . For the remaining variance, a non-shared environmental component was simulated. Effects from each locus, the polygenic variance component and the non-shared environmental component were summed to create the quantitative trait (i.e., no interaction effects). In total, there were 100 trait models simulated including five single- and twotrait locus models (Additive-Null, Dominant-Null, Additive-Additive, Additive-Dominant, and Dominant-Dominant) by five levels of residual sibling correlation by four levels of ascertainment.

Simulations of non-normally distributed data were generated using the method of Bartlett and Vieland [26]. Briefly, a random deviate for the genetic component is simulated assuming normality but is then converted to a kurtotic distribution by using the cumulative distribution function of the simulated component and then calculating the inverse cumulative distribution function for, in this case, a t distribution with 12 d.f., and finally was standardized back to the $\mu=0, \sigma=1$ scale prior to analysis with the PPL. This procedure has the advantage that the non-normal data can be compared to the normal data since the two are always proportional for each individual in the data set, making correlation of results from analyses of the normal and non-normal data sets directly interpretable.

\section{Censoring and Analysis Procedures}

The censoring scenario we created was based on the assumption that 'affected' individuals would have a treatment effect, thus necessitating removal of those QT values from the analysis. Assuming the ascertainment threshold in the simulations is the same as the diagnosis threshold of a treatable disease, the presence of extreme trait values in the simulated data indicates which family members will be censored. Any trait value greater than the ascertainment criteria was set to unknown (missing data). This procedure also ensures that every pedigree will have at least one censored data point since every pedigree was required to have at least one child with a trait value above the ascertainment threshold.

To assess performance of the QTT model, we compared the PPL using the QTT model on the censored data to the QT model using the original simulated data (no censoring). This comparison provides information on how well the QTT model recovers the missing information. We additionally compared the QT model on the original simulated data and data where the censored observation was fixed to a 'sensible constant'. For VCA, we used SOLAR version 4.3.1, correcting for ascertainment by fixing the trait mean and standard deviation to their corresponding simulated population values [30]. MERLIN-REGRESS statistic [28] was calculated with MELRIN v1.1.2 [31]. To quantify the change in a given test statistic, the absolute (value) percent change from the baseline analysis was computed as $\left|x_{\text {baseline }}-x_{\text {imputed }}\right| / x_{\text {baseline }}$.

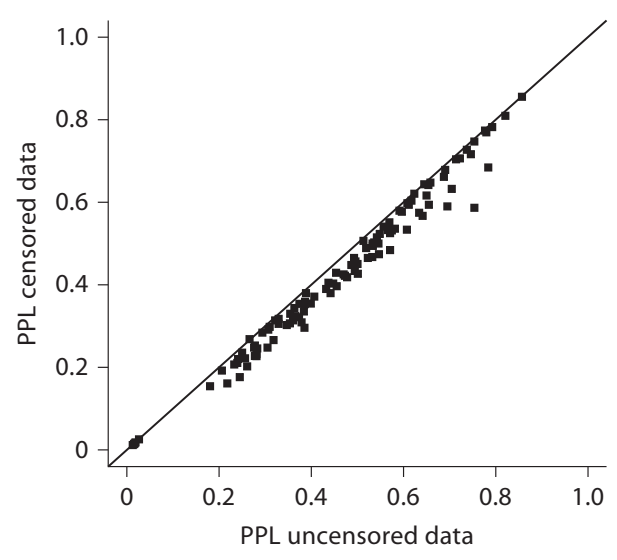

Fig. 1. PPL of the QT model on uncensored data versus PPL of the QTT model on censored data. The threshold model shows only a slight decrease in the average PPL across all genetic models and all sample ascertainment schemes. The scatter plot compares the average PPL for each generating condition (single- or two-locus by additive or dominant by RFC) comparing performance under the QT model (x-axis), which uses quantitative phenotypes on all subjects, versus the QTT model (y-axis), which uses the threshold model to integrate over a single censored subject.

\section{Results}

We will discuss the performance of the QTT model for censored data in this section, always comparing results of a mathematical or simulated data manipulation to a baseline or control condition. The key metrics of performance are changes in the average PPL across simulated generating models but within a testing condition, and the correlation in the average PPLs.

On collections of randomly ascertained families, the PPL performs as expected with all phenotypes known in the analysis (table 1, QT model on the left), recapitulating previous PPL results [26]. As in that study, there is a clear dependence of the average PPL on RFC similar to what is documented in VC methods [32, 33], where models with greater RFC are consistently associated with higher average PPLs. However, this dependence is reversed across ascertainment schemes as ascertainment criteria become more extreme (table 1, top to bottom). Ending with ASC 3 models, the average PPL decreases with increasing familial correlation. Application of the QTT model (table 1, QTT model on the right) does not alter any of the aforementioned observations. Though the average PPL is lower in all cases reflecting the loss of phenotypic information due to censoring (fig. 1), the reduction in the average PPL is slight. 
Table 1. Average PPL (SD) for the simulation generating models

\begin{tabular}{|c|c|c|c|c|c|c|c|}
\hline \multirow[t]{2}{*}{ Ascertainment } & \multicolumn{3}{|l|}{ Model } & \multicolumn{2}{|l|}{ QT } & \multicolumn{2}{|l|}{ QTT } \\
\hline & locus 1 & locus 2 & RFC & locus 1 & locus 2 & locus 1 & locus 2 \\
\hline \multirow[t]{25}{*}{ Random* } & Add & Null & 0.0 & $0.14(0.21)$ & $0.01(0.01)$ & & \\
\hline & & & 0.1 & $0.15(0.20)$ & $0.01(0.03)$ & & \\
\hline & & & 0.2 & $0.20(0.25)$ & $0.01(0.05)$ & & \\
\hline & & & 0.3 & $0.27(0.30)$ & $0.02(0.07)$ & & \\
\hline & & & 0.4 & $0.39(0.34)$ & $0.02(0.06)$ & & \\
\hline & $\overline{\text { Add }}$ & Add & 0.0 & $0.14(0.21)$ & $0.14(0.23)$ & & \\
\hline & & & 0.1 & $0.20(0.26)$ & $0.18(0.26)$ & & \\
\hline & & & 0.2 & $0.26(0.30)$ & $0.25(0.29)$ & & \\
\hline & & & 0.3 & $0.37(0.34)$ & $0.37(0.32)$ & & \\
\hline & & & 0.4 & $0.52(0.38)$ & $0.58(0.34)$ & & \\
\hline & $\overline{\text { Add }}$ & Dom & 0.0 & $0.15(0.22)$ & $0.18(0.24)$ & & \\
\hline & & & 0.1 & $0.18(0.25)$ & $0.26(0.31)$ & & \\
\hline & & & 0.2 & $0.24(0.28)$ & $0.36(0.34)$ & & \\
\hline & & & 0.3 & $0.34(0.32)$ & $0.52(0.35)$ & & \\
\hline & & & 0.4 & $0.49(0.36)$ & $0.74(0.29)$ & & \\
\hline & $\overline{\text { Dom }}$ & Null & 0.0 & $0.23(0.30)$ & $0.01(0.01)$ & & \\
\hline & & & 0.1 & $0.25(0.31)$ & $0.01(0.02)$ & & \\
\hline & & & 0.2 & $0.30(0.33)$ & $0.01(0.02)$ & & \\
\hline & & & 0.3 & $0.39(0.36)$ & $0.01(0.03)$ & & \\
\hline & & & 0.4 & $0.54(0.36)$ & $0.01(0.03)$ & & \\
\hline & $\overline{\text { Dom }}$ & Dom & 0.0 & $0.16(0.23)$ & $0.18(0.25)$ & & \\
\hline & & & 0.1 & $0.23(0.29)$ & $0.23(0.27)$ & & \\
\hline & & & 0.2 & $0.30(0.33)$ & $0.31(0.31)$ & & \\
\hline & & & 0.3 & $0.41(0.36)$ & $0.45(0.34)$ & & \\
\hline & & & 0.4 & $0.59(0.36)$ & $0.66(0.33)$ & & \\
\hline \multirow[t]{25}{*}{$1 \mathrm{SD}$} & Add & Null & 0.0 & $0.25(0.29)$ & $0.01(0.03)$ & $0.23(0.28)$ & $0.01(0.03)$ \\
\hline & & & 0.1 & $0.24(0.30)$ & $0.01(0.01)$ & $0.22(0.30)$ & $0.01(0.02)$ \\
\hline & & & 0.2 & $0.28(0.32)$ & $0.01(0.01)$ & $0.23(0.30)$ & $0.01(0.01)$ \\
\hline & & & 0.3 & $0.28(0.32)$ & $0.01(0.01)$ & $0.25(0.31)$ & $0.01(0.01)$ \\
\hline & & & 0.4 & $0.36(0.33)$ & $0.02(0.01)$ & $0.32(0.33)$ & $0.01(0.01)$ \\
\hline & $\overline{\text { Add }}$ & Add & 0.0 & $0.26(0.30)$ & $0.21(0.26)$ & $0.22(0.27)$ & $0.19(0.27)$ \\
\hline & & & 0.1 & $0.28(0.31)$ & $0.24(0.29)$ & $0.25(0.29)$ & $0.21(0.27)$ \\
\hline & & & 0.2 & $0.26(0.28)$ & $0.25(0.28)$ & $0.22(0.25)$ & $0.24(0.28)$ \\
\hline & & & 0.3 & $0.32(0.34)$ & $0.35(0.33)$ & $0.27(0.31)$ & $0.33(0.33)$ \\
\hline & & & 0.4 & $0.38(0.32)$ & $0.38(0.34)$ & $0.34(0.33)$ & $0.30(0.32)$ \\
\hline & Add & Dom & 0.0 & $0.23(0.29)$ & $0.49(0.38)$ & $0.21(0.28)$ & $0.46(0.39)$ \\
\hline & & & 0.1 & $0.18(0.26)$ & $0.58(0.37)$ & $0.15(0.21)$ & $0.53(0.37)$ \\
\hline & & & 0.2 & $0.27(0.30)$ & $0.61(0.35)$ & $0.27(0.31)$ & $0.53(0.35)$ \\
\hline & & & 0.3 & $0.25(0.28)$ & $0.69(0.33)$ & $0.24(0.28)$ & $0.59(0.35)$ \\
\hline & & & 0.4 & $0.28(0.32)$ & $0.78(0.29)$ & $0.23(0.27)$ & $0.68(0.34)$ \\
\hline & Dom & Null & 0.0 & $0.58(0.38)$ & $0.01(0.02)$ & $0.54(0.37)$ & $0.01(0.02)$ \\
\hline & & & 0.1 & $0.65(0.35)$ & $0.01(0.01)$ & $0.62(0.37)$ & $0.01(0.01)$ \\
\hline & & & 0.2 & $0.63(0.35)$ & $0.01(0.01)$ & $0.57(0.38)$ & $0.02(0.04)$ \\
\hline & & & 0.3 & $0.65(0.34)$ & $0.01(0.01)$ & $0.59(0.36)$ & $0.01(0.01)$ \\
\hline & & & 0.4 & $0.75(0.30)$ & $0.01(0.01)$ & $0.59(0.36)$ & $0.01(0.01)$ \\
\hline & Dom & Dom & 0.0 & $0.41(0.35)$ & $0.45(0.38)$ & $0.37(0.34)$ & $0.40(0.37)$ \\
\hline & & & 0.1 & $0.44(0.34)$ & $0.49(0.36)$ & $0.40(0.34)$ & $0.43(0.35)$ \\
\hline & & & 0.2 & $0.50(0.36)$ & $0.55(0.37)$ & $0.45(0.36)$ & $0.50(0.39)$ \\
\hline & & & 0.3 & $0.57(0.35)$ & $0.64(0.35)$ & $0.48(0.35)$ & $0.57(0.36)$ \\
\hline & & & 0.4 & $0.64(0.34)$ & $0.70(0.31)$ & $0.57(0.37)$ & $0.63(0.36)$ \\
\hline
\end{tabular}


Table 1 (continued)

\begin{tabular}{|c|c|c|c|c|c|c|c|}
\hline \multirow[t]{2}{*}{ Ascertainment } & \multicolumn{3}{|l|}{ Model } & \multicolumn{2}{|l|}{ QT } & \multicolumn{2}{|l|}{ QTT } \\
\hline & locus 1 & locus 2 & RFC & locus 1 & locus 2 & locus 1 & locus 2 \\
\hline \multirow[t]{25}{*}{$2 \mathrm{SD}$} & Add & Null & 0.0 & $0.61(0.34)$ & $0.01(0.01)$ & $0.60(0.34)$ & $0.01(0.01)$ \\
\hline & & & 0.1 & $0.54(0.37)$ & $0.01(0.01)$ & $0.52(0.38)$ & $0.01(0.01)$ \\
\hline & & & 0.2 & $0.49(0.38)$ & $0.01(0.01)$ & $0.47(0.38)$ & $0.01(0.01)$ \\
\hline & & & 0.3 & $0.45(0.34)$ & $0.02(0.02)$ & $0.43(0.35)$ & $0.02(0.02)$ \\
\hline & & & 0.4 & $0.44(0.34)$ & $0.02(0.02)$ & $0.41(0.34)$ & $0.02(0.02)$ \\
\hline & $\overline{\text { Add }}$ & Add & 0.0 & $0.36(0.31)$ & $0.39(0.33)$ & $0.34(0.30)$ & $0.38(0.32)$ \\
\hline & & & 0.1 & $0.39(0.35)$ & $0.32(0.33)$ & $0.38(0.36)$ & $0.31(0.32)$ \\
\hline & & & 0.2 & $0.31(0.30)$ & $0.31(0.32)$ & $0.30(0.31)$ & $0.30(0.32)$ \\
\hline & & & 0.3 & $0.37(0.35)$ & $0.31(0.30)$ & $0.35(0.34)$ & $0.29(0.31)$ \\
\hline & & & 0.4 & $0.36(0.32)$ & $0.39(0.35)$ & $0.32(0.32)$ & $0.35(0.35)$ \\
\hline & $\overline{\text { Add }}$ & Dom & 0.0 & $0.39(0.33)$ & $0.65(0.35)$ & $0.36(0.33)$ & $0.64(0.36)$ \\
\hline & & & 0.1 & $0.33(0.33)$ & $0.66(0.33)$ & $0.31(0.32)$ & $0.65(0.33)$ \\
\hline & & & 0.2 & $0.29(0.29)$ & $0.69(0.31)$ & $0.28(0.29)$ & $0.66(0.31)$ \\
\hline & & & 0.3 & $0.27(0.28)$ & $0.72(0.32)$ & $0.25(0.26)$ & $0.71(0.33)$ \\
\hline & & & 0.4 & $0.33(0.32)$ & $0.79(0.28)$ & $0.32(0.33)$ & $0.78(0.28)$ \\
\hline & Dom & Null & 0.0 & $0.78(0.33)$ & $0.01(0.01)$ & $0.77(0.33)$ & $0.01(0.01)$ \\
\hline & & & 0.1 & $0.75(0.32)$ & $0.02(0.02)$ & $0.75(0.32)$ & $0.02(0.03)$ \\
\hline & & & 0.2 & $0.78(0.32)$ & $0.02(0.02)$ & $0.77(0.32)$ & $0.02(0.03)$ \\
\hline & & & 0.3 & $0.82(0.26)$ & $0.02(0.03)$ & $0.81(0.26)$ & $0.02(0.04)$ \\
\hline & & & 0.4 & $0.86(0.25)$ & $0.03(0.09)$ & $0.86(0.25)$ & $0.03(0.09)$ \\
\hline & Dom & Dom & 0.0 & $0.66(0.35)$ & $0.64(0.33)$ & $0.65(0.35)$ & $0.64(0.33)$ \\
\hline & & & 0.1 & $0.62(0.34)$ & $0.57(0.35)$ & $0.60(0.35)$ & $0.55(0.35)$ \\
\hline & & & 0.2 & $0.60(0.37)$ & $0.62(0.35)$ & $0.59(0.37)$ & $0.62(0.36)$ \\
\hline & & & 0.3 & $0.71(0.31)$ & $0.69(0.35)$ & $0.70(0.31)$ & $0.68(0.35)$ \\
\hline & & & 0.4 & $0.75(0.31)$ & $0.74(0.33)$ & $0.72(0.32)$ & $0.73(0.34)$ \\
\hline \multirow[t]{25}{*}{$3 \mathrm{SD}$} & Add & Null & 0.0 & $0.56(0.35)$ & $0.01(0.02)$ & $0.54(0.36)$ & $0.02(0.02)$ \\
\hline & & & 0.1 & $0.52(0.37)$ & $0.01(0.01)$ & $0.49(0.36)$ & $0.02(0.01)$ \\
\hline & & & 0.2 & $0.47(0.35)$ & $0.02(0.01)$ & $0.43(0.34)$ & $0.02(0.01)$ \\
\hline & & & 0.3 & $0.37(0.34)$ & $0.02(0.01)$ & $0.32(0.32)$ & $0.02(0.01)$ \\
\hline & & & 0.4 & $0.38(0.32)$ & $0.02(0.01)$ & $0.31(0.30)$ & $0.02(0.01)$ \\
\hline & $\overline{\text { Add }}$ & Add & 0.0 & $0.55(0.37)$ & $0.53(0.36)$ & $0.52(0.36)$ & $0.50(0.36)$ \\
\hline & & & 0.1 & $0.47(0.36)$ & $0.40(0.33)$ & $0.42(0.35)$ & $0.35(0.32)$ \\
\hline & & & 0.2 & $0.36(0.35)$ & $0.36(0.32)$ & $0.31(0.34)$ & $0.31(0.31)$ \\
\hline & & & 0.3 & $0.28(0.30)$ & $0.26(0.29)$ & $0.23(0.27)$ & $0.20(0.26)$ \\
\hline & & & 0.4 & $0.24(0.25)$ & $0.22(0.25)$ & $0.18(0.21)$ & $0.16(0.22)$ \\
\hline & $\overline{\text { Add }}$ & Dom & 0.0 & $0.54(0.37)$ & $0.59(0.40)$ & $0.51(0.37)$ & $0.58(0.40)$ \\
\hline & & & 0.1 & $0.49(0.36)$ & $0.53(0.35)$ & $0.45(0.35)$ & $0.50(0.35)$ \\
\hline & & & 0.2 & $0.35(0.33)$ & $0.49(0.34)$ & $0.30(0.31)$ & $0.44(0.33)$ \\
\hline & & & 0.3 & $0.30(0.30)$ & $0.48(0.35)$ & $0.25(0.27)$ & $0.42(0.34)$ \\
\hline & & & 0.4 & $0.28(0.31)$ & $0.53(0.38)$ & $0.23(0.29)$ & $0.47(0.38)$ \\
\hline & Dom & Null & 0.0 & $0.51(0.37)$ & $0.01(0.01)$ & $0.51(0.37)$ & $0.02(0.01)$ \\
\hline & & & 0.1 & $0.61(0.38)$ & $0.02(0.02)$ & $0.59(0.38)$ & $0.02(0.02)$ \\
\hline & & & 0.2 & $0.57(0.34)$ & $0.01(0.01)$ & $0.53(0.35)$ & $0.02(0.01)$ \\
\hline & & & 0.3 & $0.57(0.35)$ & $0.02(0.01)$ & $0.53(0.35)$ & $0.02(0.01)$ \\
\hline & & & 0.4 & $0.55(0.35)$ & $0.02(0.01)$ & $0.47(0.34)$ & $0.02(0.01)$ \\
\hline & Dom & Dom & 0.0 & $0.60(0.35)$ & $0.55(0.36)$ & $0.58(0.35)$ & $0.52(0.36)$ \\
\hline & & & 0.1 & $0.53(0.37)$ & $0.49(0.35)$ & $0.50(0.36)$ & $0.46(0.36)$ \\
\hline & & & 0.2 & $0.53(0.36)$ & $0.43(0.31)$ & $0.49(0.36)$ & $0.39(0.30)$ \\
\hline & & & 0.3 & $0.52(0.36)$ & $0.45(0.34)$ & $0.47(0.36)$ & $0.40(0.33)$ \\
\hline & & & 0.4 & $0.50(0.35)$ & $0.44(0.36)$ & $0.43(0.35)$ & $0.38(0.35)$ \\
\hline
\end{tabular}

RFC $=$ Residual familial correlation (heritability not accounted for by either locus). ${ }^{*}$ Random ascertainment is population-based data with no ascertainment threshold. These data are listed as a gold standard baseline for further comparisons. 
Fig. 2. Comparison of analysis of censored data by various methods. a-c Censored data are imputed using Tobin method. Each dot in the scatter plots represents the average value of the test statistics for one generating condition. In all cases, analysis with the censored data (y-axis) is compared to the analysis of the same data without censoring (x-axis). The Tobin method may be conservative when using regression analysis (a), and either conservative or anti-conservative when applied in VCA (b). The trends are associated with the ascertainment scheme. The Tobin method does induce a decrease in the average PPL for the ASC 1 condition (c), which is mitigated by using the threshold model (d).
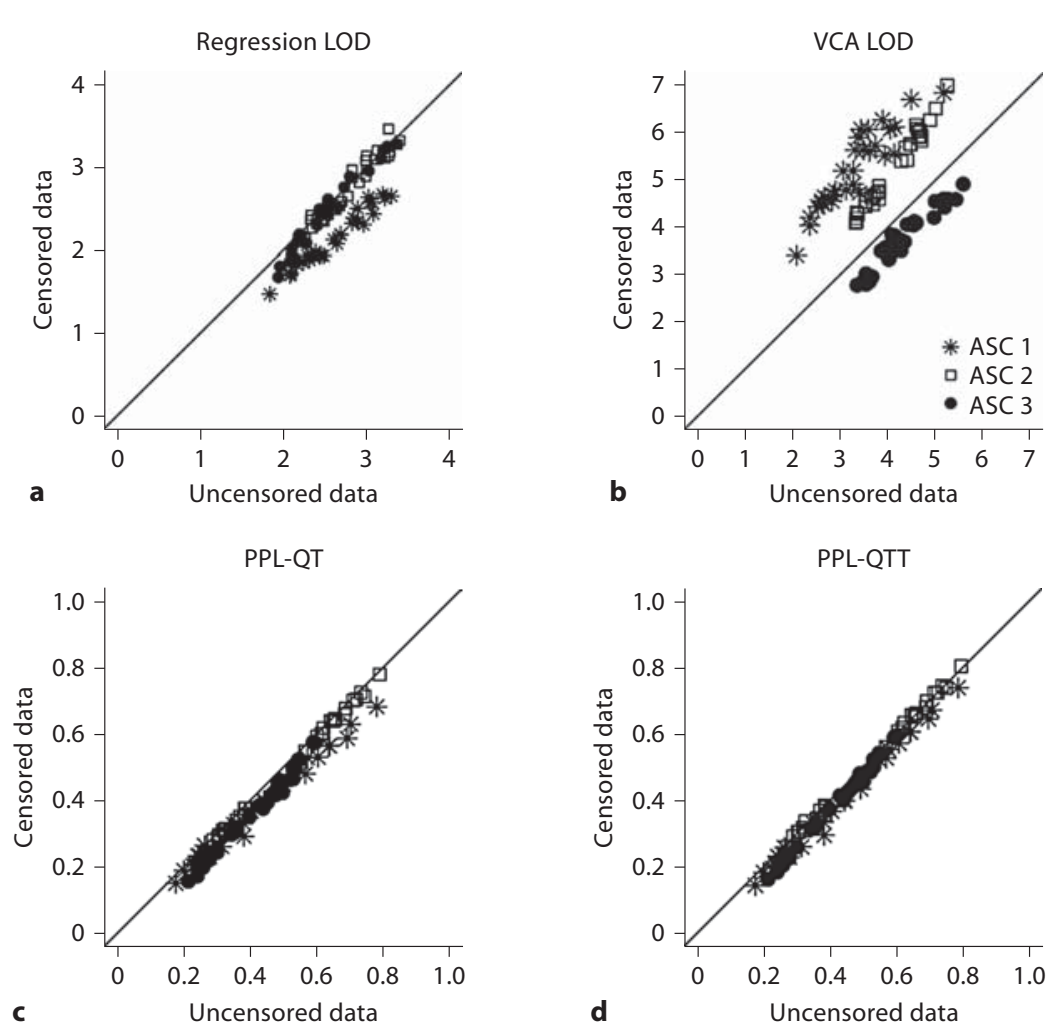

PPL-QTT

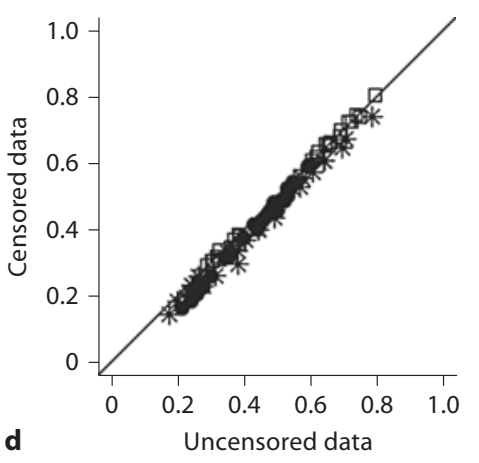

We contrast the QTT model with the ad hoc 'sensible constant' approach [1], whereby data missing due to censoring are imputed with a constant selected to be representative of all missing data based on a priori assumptions alone. We applied this procedure, choosing a constant of $3 \mathrm{SD}$ above the population mean. Regression shows a deflation of the test statistic with censored data (fig. 2a). Depending upon the ascertainment condition, VCA (fig. 2b) shows either deflation or inflation of the test statistic, whereby deflation occurs at ASC 3, but inflation occurs at ASC 1 and ASC 2.

For the PPL, the sensible constant procedure shows a high correlation between the QT model without censoring and the use of a sensible constant model (fig. 2c); however, figure $2 \mathrm{~d}$ indicates that, on average, the QTT model performs better. The absolute (value) percent change from the baseline analysis to the analysis with the sensible constant was an $8.7 \%$ change in the PPL statistic, while regression had an absolute (value) percent change from the baseline of $12.5 \%$ and VCA an average change of $58.9 \%$. By way of contrast, the QTT model had an ab- solute (value) percent change from the baseline analysis of only $6.8 \%$.

The QT model within the PPL framework was shown to be robust to deviations from normality [26] when analyzing quantitative traits, specifically when testing excess kurtosis. Similar testing of the QTT model indicates that it also inherits this property (fig. 3a). Analysis of matched normal/non-normal data (see Methods section) shows essentially no change in the average PPL, though as before there is a decrease in the average PPL when using the QTT model, consistent with the reduction in phenotypic information from censoring, but non-normality does not appear to have a systematic effect on the robustness of the PPL using the QTT model. As a control condition, to show that the simulated non-normal data were suitably extreme to be a valid test of the PPL's sensitivity to nonnormality, the same data were analyzed with VCA and pedigree-based regression. VCA shows marked inflation (fig. 3b), indicating the simulated data were suitably nonnormal to be a fair test of the PPL QTT model. Pedigreebased regression also shows inflation of the test statistic 
Fig. 3. Plot of effect of non-normality on PPL and VCA under all generating conditions. a The average PPL for the QTT model under each generating condition is plotted for normal data (x-axis) versus nonnormal data (y-axis). The threshold model is not affected by non-normally distributed data. $\mathbf{b}$ The average LOD score for VCA under each generating condition is plotted for normal data (x-axis) versus non-normal data (y-axis). As expected, there is a large inflationary effect for non-normal data analyzed by VCA.

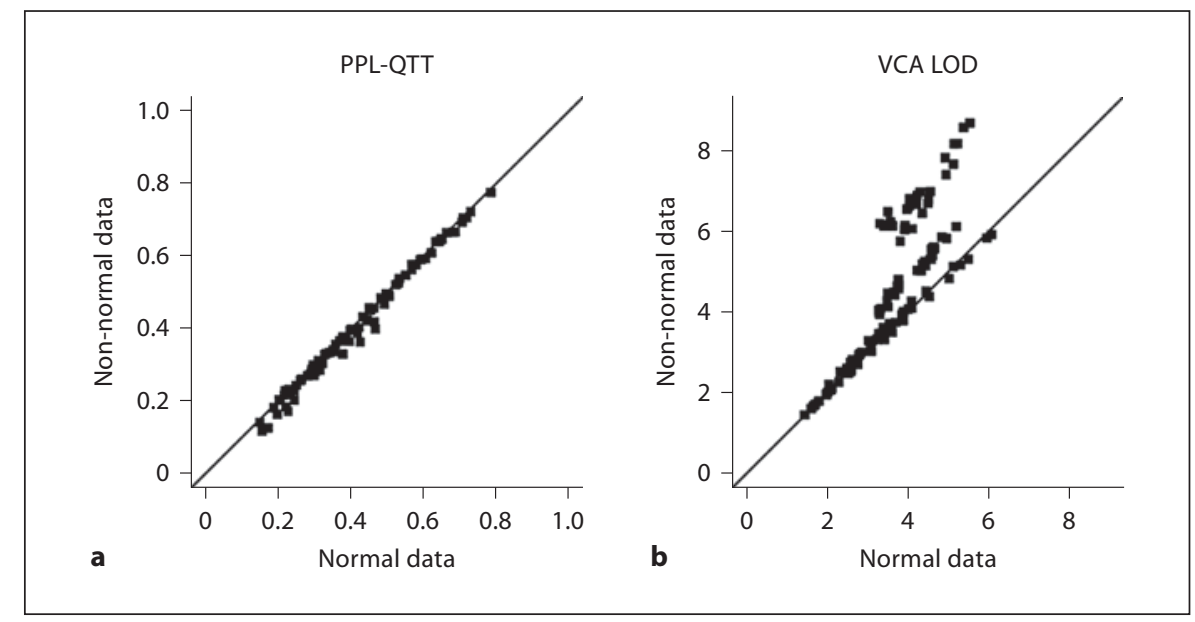

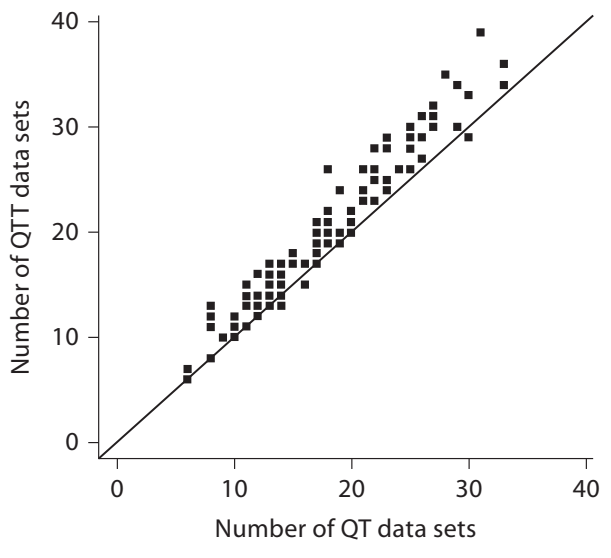

Fig. 4. The QTT model accumulates evidence for linkage similar to the QT model as more data sets are added into the analysis. The number of simulated data sets added required to attain a PPL of 1 using Bayesian sequential updating was measured on the uncensored data using the QT model (x-axis) and the censored data using the QTT model (y-axis). The average number of replicates for each model is plotted. The QTT model requires $0-3$ additional data sets to achieve a PPL of 1 relative to the QT model, consistent with the slightly lower average PPL in the QTT model.

(data not shown) but is known to be more robust to violations of the normality assumption.

The signature feature of the PPL framework is the flexibility to add in new data and accumulate evidence through sequentially updating across data sets. This procedure increases sample size without assuming homogeneity, within or between data sets, since the PPL is based on the admixture likelihood and model integration occurs within each data set locally. Only the posterior distribution of the parameter theta (i.e., the linkage information) is updated across data sets. The PPL has been proven to converge to 1 as more data are collected when linkage exists and to converge to 0 when linkage is not true [24]. Since this convergence property is not expected to be affected by the inclusion of additional trait parameters, we assessed if the convergence was adversely affected by the addition of the threshold parameter. Within each generating condition, one replicate data set was randomly chosen to be the 'initial' data set, and additional replicate data sets were sampled without replacement for sequential updating with the PPL from the last round of updating. The outcome variable is the number of data sets required to achieve a PPL of 1 when the data were generated assuming linkage. Figure 4 compares the rate of PPL convergence to 1 for the QT model on the full data versus the QTT model on the censored data. For all simulating conditions, the rate of convergence is virtually identical for the QT and QTT models, but analysis of censored data using the QTT model is slightly less efficient, as expected, since on average the PPL under the QTT model is slightly less than that under the QT model.

\section{Discussion}

We have evaluated the QTT model that handles a fundamental complexity of real-world data; specifically, we examined missing phenotypes due to censoring when the missing data can be reasonably inferred to be above or below an unknown threshold. As hypothesized, the ad- 
Fig. 5. The effect of RFC on the average PPL by genetic model for randomly ascertained families (a) and families ascertained for the presence of at least one child with a quantitative trait value greater than 3 SD above the population mean (b).

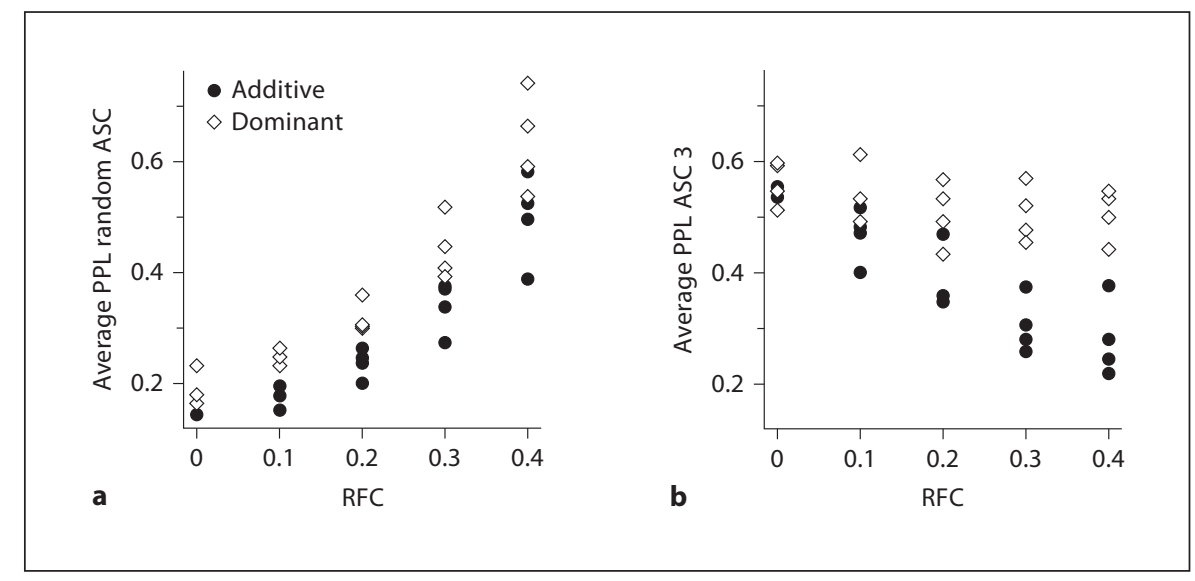

dition of a threshold parameter to the PPL framework appears to have little effect on the desirable properties of the PPL, while adding a property for analysis of censored data was shown to be advantageous compared to alternative methods. The data demonstrate that the patterns of average QTT PPL values are highly similar to those of QT PPL values across widely disparate generating conditions, including dominance and a range of RFCs, and also when analyzing non-normally distributed data. Additionally, including the threshold in the PPL shows the expected convergence to 1 when linkage is present and not when linkage is absent, thus empirically demonstrating that the sequential updating feature of the PPL framework is still available under the QTT model. Although not explicitly tested, the data also show that when pedigrees have been selected on the basis of an extreme trait, the PPL does not require additional information in the form of an ascertainment correction to capitalize on the sampling method, and this also remains true when considering the analysis with a threshold parameter.

The models we tested allow us to directly compare the QTT model applied to a data set with $>20 \%$ missing data (at least one censored observation per 5-person nuclear family) to one where the missing data point is known (where no model of censoring is needed). Overall, the average PPL decreases slightly when using the QTT model, with a change of $6.8 \%$ from the value of the QT PPL, on average, which reflects the fact that there is less phenotypic information in the analysis of the censored data (i.e., there are missing data points in the QTT model data sets that are known in the control data set). Preventing steep reductions in the average PPL due to a $>20 \%$ reduction in the sample's information rests on the assumption that missing data can be inferred to be above or below the threshold. Inaccurately inferring the direction of censoring, i.e., assuming the value should be above the threshold parameter $t$ when this is not true, would be expected to reduce the performance of the model. However, for the types of systematic censoring involving treatment effects discussed in this paper, our directionality assumption is quite reasonable as, for example, treatment effects are the result of medical interventions designed specifically for one tail of the phenotypic distribution.

We also compared the PPL threshold analysis model with an ad hoc procedure where missing data are imputed with a sensible constant that is ideally located in the truncated distribution to minimize error in the linkage statistic, though the ideal value for the sensible constant can never be known in practice. While it is always possible that a better choice for the sensible constant exists and that such a choice would perform better than the analyses we presented, the QTT model does not require choosing a sensible constant and thus circumvents the issue entirely. Our study shows an additional complication of the sensible constant procedure, i.e., that performance of the sensible constant is not uniform across all statistical methods applied in our study, with VCA being more greatly affected than the regression model. Therefore, the choice of the analysis method when using a sensible constant is a factor that must be carefully weighed to limit reductions in statistical evidence.

It was previously shown for VCA and score statistics [32] that under random ascertainment, increasing sibling correlation increases power, as also shown here for all simulating generating models (fig. 5a). Additional analytical work indicated the same increase in power holds for single ascertainment [33], where the probability of more than one proband is zero. However, under our as- 
certainment scheme that requires at least one affected child and does permit multiple probands, higher residual sibling correlation seems to reduce power (fig. 5b). We postulate that this is due to parents being indirectly ascertained for a tendency to be homozygous at the trait locus and, therefore, uninformative for linkage. This indirect selection occurs since mating types with one or more homozygous parents are more likely to produce multiple homozygous children with extreme phenotypes. Power loss is larger under the additive models than under the dominant models since dominant models exert less impact on the parents' genotypes. Importantly, the QT and QTT models are always highly positively correlated, and incorporation of the threshold parameter, the main focus of our study, is not related to this phenomena. We also observed this pattern using censored and uncensored data, so this is not a property of censoring the observed trait distribution per se.

This study is limited both by the scope of the work and the assumptions herein. To utilize the proposed method on censored data, the investigator must know something about the missing data point. The key development here is that it is not necessary to know the approximate average value of the censored data points (to create a sensible constant); the researchers only need to know on which side of the threshold the data points will reside. In practice, the integration boundaries of $t$ may be chosen conservatively to span a greater range than is even biologically permissible, provided it does not extend over all possible values of the quantitative trait. Further, while the PPL is robust to violations of normality in the trait distribution, we have only examined symmetric non-normal distributions since varying dispersion is a simple situation that is of considerable concern due to its association with increased false-positive rates in both VC and regression-based analysis. Other non-symmetric distributional forms may require additional deliberation when applying and interpreting the PPL with a threshold.

The QTT model for handling censored data is currently limited to univariate applications. However, it is reasonable to pursue more advanced phenotypic models where correlated multivariate traits have some observations censored and others not. Many multivariate methods require fully observed data for each subject to be included in the calculations, and application of the QTT model to a multivariate context would remove this restriction.

\section{Acknowledgements}

The authors would like to thank Dr. Veronica Vieland for helpful comments on an earlier version of the manuscript. We appreciate our anonymous reviewers' enthusiasm for our work, and especially the meticulous efforts of one reviewer who provided numerous suggestions that materially improved this paper. We gratefully acknowledge NIH funding from R01DC009453 (C.W.B.) and RC1MH088288 (L.H. and C.W.B.). This work was also supported in part by an allocation of computing time from the Ohio Supercomputer Center Grant PCCR0001-2 (C.W.B.).

\section{References}

1 Tobin MD, Sheehan NA, Scurrah KJ, Burton PR: Adjusting for treatment effects in studies of quantitative traits: antihypertensive therapy and systolic blood pressure. Stat Med 2005;24:2911-2935.

-2 Sillanpaa MJ, Hoti F: Mapping quantitative trait loci from a single-tail sample of the phenotype distribution including survival data. Genetics 2007;177:2361-2377.

3 Li M, Boehnke M, Abecasis GR, Song PX: Quantitative trait linkage analysis using Gaussian copulas. Genetics 2006;173:23172327.

4 Diao G, Lin DY: Semiparametric variancecomponent models for linkage and association analyses of censored trait data. Genet Epidemiol 2006;30:570-581.
Anderson CA, McRae AF, Visscher PM: A simple linear regression method for quantitative trait loci linkage analysis with censored observations. Genetics 2006;173:17351745.

6 Diao G, Lin DY: Semiparametric methods for mapping quantitative trait loci with censored data. Biometrics 2005;61:789-798.

7 Epstein MP, Lin X, Boehnke M: A tobit variance-component method for linkage analysis of censored trait data. Am J Hum Genet 2003;72:611-620.

8 Palmer LJ, Tiller KJ, Burton PR: Genomewide linkage analysis using genetic variance components of alcohol dependency-associated censored and continuous traits. Genet Epidemiol 1999;17(suppl 1):S283-S288.

$\checkmark 9$ Cui JS, Hopper JL, Harrap SB: Antihypertensive treatments obscure familial contributions to blood pressure variation. Hypertension 2003;41:207-210.
0 Puppala S, Coletta DK, Schneider J, Hu SL, Farook VS, Dyer TD, Arya R, Blangero J, Duggirala R, DeFronzo RA, Jenkinson CP: Genome-wide linkage screen for systolic blood pressure in the Veterans Administration Genetic Epidemiology Study (VAGES) of Mexican-Americans and confirmation of a major susceptibility locus on chromosome 6q14.1. Hum Hered 2011;71:1-10.

11 Schafer JL: Multiple imputation: a primer. Stat Methods Med Res 1999;8:3-15.

12 Donders AR, van der Heijden GJ, Stijnen T, Moons KG: Review: a gentle introduction to imputation of missing values. J Clin Epidemiol 2006;59:1087-1091.

13 Bartlett CW, Vieland VJ: Two novel quantitative trait linkage analysis statistics based on the posterior probability of linkage: application to the COGA families. BMC Genet 2005;6(suppl 1):S121. 
14 Wright S: An analysis of variability in the number of digits in an inbred strain of guinea pigs. Genetics 1934;19:506-536.

15 Vieland VJ, Huang Y, Seok SC, Burian J, Catalyurek U, O'Connell J, Segre A, Valentine-Cooper W: KELVIN: a software package for rigorous measurement of statistical evidence in human genetics. Hum Hered 2011;72:276-288.

16 Bartlett CW, Flax JF, Logue MW, Smith BJ, Vieland VJ, Tallal P, Brzustowicz LM: Examination of potential overlap in autism and language loci on chromosomes 2, 7, and 13 in two independent samples ascertained for specific language impairment. Hum Hered 2004;57:10-20.

17 Vieland VJ, Hallmayer J, Huang Y, Pagnamenta AT, Pinto D, Khan H, Monaco AP, Paterson AD, Scherer SW, Sutcliffe JS, Szatmari P: Novel method for combined linkage and genome-wide association analysis finds evidence of distinct genetic architecture for two subtypes of autism. J Neurodev Disord 2011;3:113-123.

18 Simmons TR, Flax JF, Azaro MA, Hayter JE, Justice LM, Petrill SA, Bassett AS, Tallal P, Brzustowicz LM, Bartlett CW: Increasing genotype-phenotype model determinism: application to bivariate reading/language traits and epistatic interactions in languageimpaired families. Hum Hered 2010;70:232244.
19 Wratten NS, Memoli H, Huang Y, Dulencin AM, Matteson PG, Cornacchia MA, Azaro MA, Messenger J, Hayter JE, Bassett AS, Buyske S, Millonig JH, Vieland VJ, Brzustowicz LM: Identification of a schizophreniaassociated functional noncoding variant in NOS1AP. Am J Psychiatry 2009; 166:434441.

20 Vieland VJ: Bayesian linkage analysis, or: how I learned to stop worrying and love the posterior probability of linkage. Am J Hum Genet 1998;63:947-954.

21 Bartlett CW, Flax JF, Logue MW, Vieland VJ, Bassett AS, Tallal P, Brzustowicz LM: A major susceptibility locus for specific language impairment is located on 13q21. Am J Hum Genet 2002;71:45-55.

22 Logue MW, Vieland VJ, Goedken RJ, Crowe RR: Bayesian analysis of a previously published genome screen for panic disorder reveals new and compelling evidence for linkage to chromosome 7. Am J Med Genet B Neuropsychiatr Genet 2003;121B:95-99.

23 Vieland VJ, Wang K, Huang J: Power to detect linkage based on multiple sets of data in the presence of locus heterogeneity: comparative evaluation of model-based linkage methods for affected sib pair data. Hum Hered 2001;51:199-208.

24 Wang K, Huang J, Vieland VJ: The consistency of the posterior probability of linkage. Ann Hum Genet 2000;64:533-553.

25 Ott J: Analysis of Human Genetic Linkage, ed 3. Baltimore, Johns Hopkins University Press, 1999.
26 Bartlett CW, Vieland VJ: Accumulating quantitative trait linkage evidence across multiple datasets using the posterior probability of linkage. Genet Epidemiol 2007;31: 91-102.

27 Allison DB, Neale MC, Zannolli R, Schork NJ, Amos CI, Blangero J: Testing the robustness of the likelihood-ratio test in a variancecomponent quantitative-trait loci-mapping procedure. Am J Hum Genet 1999;65:531544.

28 Sham PC, Purcell S, Cherny SS, Abecasis GR: Powerful regression-based quantitative-trait linkage analysis of general pedigrees. Am J Hum Genet 2002;71:238-253.

29 Vieland VJ, Huang Y, Bartlett C, Davies TF, Tomer Y: A multilocus model of the genetic architecture of autoimmune thyroid disorder, with clinical implications. Am J Hum Genet 2008;82:1349-1356.

30 Almasy L, Blangero J: Multipoint quantitative-trait linkage analysis in general pedigrees. Am J Hum Genet 1998;62:1198-1211.

31 Abecasis GR, Cherny SS, Cookson WO, Cardon LR: Merlin - rapid analysis of dense genetic maps using sparse gene flow trees. Nat Genet 2002;30:97-101.

-32 Tang HK, Siegmund D: Mapping quantitative trait loci in oligogenic models. Biostatistics 2001;2:147-162.

33 Peng J, Siegmund D: Mapping quantitative traits with random and with ascertained sibships. Proc Natl Acad Sci USA 2004;101: 7845-7850. 